ORIGINAL ARTICLE

\title{
Impaired parasympathetic response to feeding in ventilated preterm babies
}

\author{
S L Smith, A K Doig, W N Dudley
}

See end of article for authors' affiliations

Correspondence to: Sandra L Smith, College of Nursing, University of Utah, 10 South 2000 East, Salt Lake City, UT 84112, USA; Sandra.smith@nurs. utah.edu

Accepted 20 May 2005 Published Online First 7 June 2005
Background: Premature very low birthweight (VLBW) infants are born with an underdeveloped parasympathetic nervous system (PNS) which may limit their ability to respond adequately to feeding and may limit their capacities for extrauterine growth and development.

Objectives: To describe the patterns of autonomic response to feeding and identify relationships between change in heart period variability measures over time with selected infant characteristics.

Methods: Individual growth curve analysis techniques were used to describe the patterns of change over time in sympathetic and parasympathetic tone as measured by low and high frequency heart period power.

Results: Sixteen mechanically ventilated VLBW infants with a mean corrected gestational age of 30.4 weeks participated in the study. The low frequency (LF) power slope was $-17.67(p=0.0002)$ and the high frequency (HF) power slope was -0.92 (0.0003). There was a significant relationship between $\mathrm{HF}$ slope and birth gestational age $(r=-0.49, \mathrm{p}=0.05)$.

Conclusions: HF power, representing primarily parasympathetic activity, did not increase with enteral feeding as anticipated. LF power, an indicator of sympathetic tone, decreased during and after feeding suggesting the anticipated effect of inhibition of the sympathetic nervous system in response to the gut stimulus. Critically ill VLBW infants possess an overriding sympathetic response, but may not have adequate PNS tone development.
A utonomic control of cardiac and other involuntary tissues is not fully developed until 37-38 weeks gestation. ${ }^{1-5}$ Studies of intrauterine activity show that the early fetus has a predominantly sympathetic nervous system (SNS) and that parasympathetic nervous system (PNS) control gradually increases with post-conceptional age. ${ }^{6}$ Fleisher et al showed that healthy fetuses demonstrated increasing heart period variability (HPV) consistent with improved SNS/PNS balance between 20 and 36 gestational weeks. ${ }^{7}$

Research on autonomic nervous system (ANS) development in the preterm infant has described interindividual HPV across gestational age groups and the corresponding ANS response to stress. In the healthy preterm infant, as with the fetus in utero, sympathetic tone predominates and parasympathetic tone gradually increases as the infant matures. ${ }^{8-10}$ One of the physiological consequences of extremely premature birth is a PNS that may not adequately respond to environmental stimuli such as feeding and may not be active enough to promote balanced homeostasis, growth, and development. It is unclear whether the patterns of ANS development in the intubated very low birthweight (VLBW) infant are similar to those observed in the fetus in utero and the healthier premature infants in the aforementioned studies.

In this study, we examined how the introduction of a parasympathetic stimulus, in this case feeding, was associated with the PNS response of intubated VLBW infants (2432 weeks corrected gestational age (CGA)). Based on previous HPV research in fetal and other premature infant populations, it was anticipated that infants of older gestational age would demonstrate improved parasympathetic response to this stimulus.

Heart rate and number of bradycardic events are general indicators of autonomic control but do not discriminate between the underlying components of the ANS. Power spectral analysis of HPV reveals two frequency regions, each under the primary influence of a separate branch of the ANS. ${ }^{11}$ The low frequency (LF) heart period power region, representing sympathetic activity with some parasympathetic influence, ranges from $0.02 \mathrm{~Hz}$ up to, but not including, $0.2 \mathrm{~Hz}$. The high frequency (HF) heart period power region, representing primarily parasympathetic activity, begins at $0.2 \mathrm{~Hz}$ and ranges up to and includes $2.0 \mathrm{~Hz} .^{12}{ }^{13}$

Examination of heart period power provides a mechanism for analysing the balance between sympathetic and parasympathetic tone. Increased sympathetic activity is often caused by physiological stress, while enhanced parasympathetic tone can indicate an increase in digestive and other parasympathetic activities. ${ }^{9}$ Promoting PNS tone in premature infants has been shown to promote growth and development, as well as recovery from illness. ${ }^{2}$ Heart period power is therefore a valuable clinical and research measure of the autonomic responses and capabilities in this critically ill infant population. Change in HPV across time, during various environmental fluctuations, may be indicative of the infant's ability to adapt. Our previous results from this sample of VLBW infants suggested that overall parasympathetic tone did not improve significantly with age while the infants were in the neonatal intensive care unit (NICU) environment. ${ }^{14}$ The goal of this analysis was to determine if the PNS of these extremely immature infants was capable of responding to a basic gut stimulus. We hypothesised that feeding would increase parasympathetic activity, as measured by an increase in HF power during and after feeding. All nasogastric tube feedings took place while the infants were in the incubator,

Abbreviations: ANS, autonomic nervous system; CGA, corrected gestational age; HF, high frequency; HPV, heart period variability; LF, low frequency; NICU, neonatal intensive care unit; PNS,

parasympathetic nervous system; SNS, sympathetic nervous system; VLBW, very low birthweight 
Table 1 Slope and intercept of low frequency and high frequency power in 16 intubated VLBW infants around feeding

\begin{tabular}{lrllll}
\hline Effect & Estimate & Error & df & $t$ & $\mathbf{p}$ \\
\hline Low frequency & & & & & \\
$\quad$ Intercept & 56.26 & 8.18 & 15 & 6.88 & 0.0001 \\
$\quad \begin{array}{l}\text { Slope } \\
\text { High frequency }\end{array}$ & -17.67 & 3.65 & 15 & -4.84 & 0.0002 \\
$\quad$ Intercept & 6.08 & 0.49 & 15 & 13.56 & 0.0001 \\
$\quad$ Slope & -0.92 & 0.20 & 16 & -4.63 & 0.0003 \\
\hline & & & & & \\
\hline
\end{tabular}

an environment that was not particularly chaotic or stressful to the infant.

In the current study, individual change over time is conceptualised as following a trajectory that reflects some underlying biological process. With more than two measurement occasions, one can estimate slope for each research participant with a statistical technique known as individual growth curve analysis. ${ }^{15-17}$ In the current study, this focus on the slope or pattern of change within the individual is consistent with a dynamic model of change in which the infant adapts (or fails to adapt) to changes in the environment, specifically feeding.

We hypothesised that the slope in the HF region of HPV would increase with stimulation of the gut by enteral feeding. The power in the HF region would be lower prior to feeding, increase with feeding, and remain elevated after feeding. Conversely, the power in the LF region, the region primarily influenced by the SNS, would be suppressed during and after feeding and thus show a negative slope. The specific aims of this study were to categorise patterns of intra-individual heart period power over time, specifically around enteral feeding, describe changes in HPV in relation to feeding, and correlate trends in HPV over time with selected infant characteristics.

\section{METHODS}

\section{Study design}

Individual growth curve analysis was performed on data collected for a study that compared infant physiological responses during maternal skin to skin holding and routine incubator care. During the incubator care phase, the infant was in the incubator and received nursing care every $3 \mathrm{~h}$ around a feed, which is the standard of care at the study site NICU. Details regarding the design and data collection protocols of the parent study are described elsewhere. ${ }^{18}$ National Institutes of Health funding was obtained to perform two secondary analyses: (i) describe normative values for heart period power across gestational age and

Table 2 Correlations of selected characteristics of 16 intubated VLBW infants with the slope of low frequency and high frequency power

\begin{tabular}{llllll}
\hline & LF slope & & & HF slope \\
\cline { 2 - 3 } \cline { 5 - 6 } Characteristic & $r$ & $\mathbf{p}$ & & & $\mathbf{p}$ \\
\hline Birth GA & -0.23 & NS & & -0.49 & 0.05 \\
Corrected GA & 0.02 & NS & & -0.32 & NS \\
Birth weight (g) & -0.29 & NS & & -0.48 & 0.06 \\
Study entry weight (g) & -0.04 & NS & & -0.29 & NS \\
Study entry DOL & 0.31 & NS & & 0.27 & NS \\
\hline \multirow{2}{*}{ DOL, day of life; GA, gestational age. } & & \\
\hline
\end{tabular}

other infant characteristics, ${ }^{14}$ and (ii) describe patterns of change in heart period power using growth curve analysis (reported in this paper). Institutional Review Board approval was obtained for all analyses and informed consent was obtained from the infants' mothers for the original protocol.

\section{Subjects}

Data from 16 intubated and mechanically ventilated VLBW infants with respiratory disease were included in this study. Heart period data acquired during maternal skin to skin holding were excluded from the secondary analysis due to significant differences when compared to routine incubator care $\left(F_{1,13}=11.15, \mathrm{p}=0.005\right)$. All infants were medically stable on the ventilator or were in the process of being weaned, and were on full enteral feedings administered by nasogastric tube. Other inclusion and exclusion criteria are described by Smith. ${ }^{18}$

\section{Heart period power}

Heart period ( $\mathrm{r}-\mathrm{r}$ interval) data were computer acquired 5 times per second in 5 min epochs scheduled around three infant feeding times throughout each $8 \mathrm{~h}$ study day. A total of 4 days of data per infant were obtained. Procedures for physiological data acquisition are described elsewhere. ${ }^{18} 19$

Power spectral analyses of heart period data were performed to extract the power within the regions of high and low frequency as described by Smith et al. ${ }^{14}$ The HF power and LF power data were then submitted to individual growth curve analyses to study individual trajectories of change over time as a way of examining the infants' response to PNS stimuli. Within this individual growth curve model of change, we anticipated that the slopes in the HF region would be positive, thus indicating an increase in PNS tone during feeding.

\section{Statistical analysis}

Individual growth curve analyses were conducted on the HF and LF power data using the processes recommended by Singer and Willett. ${ }^{20}{ }^{21}$ For the following analyses, data were aggregated into three measurement occasions: pre-feeding, feeding, and post-feeding times. HF and LF data for each infant were plotted over these three times of measurement. This exploratory technique provided a visual indication of the shape and direction of individual changes over time. For each infant, linear regression lines were fit for each of 4 days. Polynomial models for each infant were estimated in which the data were centred around the feeding time, which was the mid-point of the data. The nature of the model is as follows:

$$
\mathrm{Y}_{\mathrm{it}}=\pi_{0 \mathrm{i}}+\pi_{\mathrm{li}}[\mathrm{TIME}]_{\mathrm{it}}+\mathrm{R}_{\mathrm{it}}
$$

where $\mathrm{Y}_{\mathrm{it}}$ represents the level of HF or LF power for each individual (i) at time (t); t represents the time of measurement centred about feeding and thus took on three values, $-1,0$, and $1 ; \pi_{0 \mathrm{i}}$ represents each individual's intercept, which occurs at the feeding time point; $\pi_{\mathrm{li}}[\text { TIME }]_{\mathrm{it}}$ represents the linear rate of change over time (that is, the slope); and $\mathrm{R}_{\mathrm{it}}$ is an $\mathrm{i}$ by $\mathrm{t}$ matrix of model residuals at time $\mathrm{t}$ for individual $\mathrm{i}$.

When implemented within statistical software SAS PROC MIXED (SAS 8.02, Cary, NC), this polynomial form of linear change allows for quantification of an individual trajectory (intercept and slope) for every case in the study. An advantage of PROC MIXED is that the form of the covariance matrix of the individual residuals can be specified by the user. The unstructured form provided the best fit and was employed in the analyses reported below. In addition to the overall fit, the analyses provide individual parameters (intercept and slope for each infant) in the HF and LF power 
variables. In this study we focus on the slope. These individual slope parameters were then correlated with individual characteristics, using the Pearson product moment correlation.

\section{RESULTS}

\section{Sample characteristics}

At the time of data collection, all 16 VLBW infants were mechanically ventilated for respiratory disease of prematurity. Mean birth gestational age was 25.8 weeks (SD 1.6, range 24-29) and mean CGA age when the infants entered the study was 30.4 weeks (SD 1.5, range 28-34). Mean birth weight was $763.7 \mathrm{~g}$ (SD 160.7, range 600-1121) and mean weight at study entry was $983.8 \mathrm{~g}$ (SD 209.7, range 7631409).

\section{Individual growth curve analysis}

Two individual growth curve models were fit to the LF and HF power data. The slopes of HF power and LF power were negative, and both were significantly different from zero. The negative slope of LF power was much steeper than that of HF power, which was relatively flat. The results from these models are shown in table 1.

There were no significant correlations between selected infant characteristics and the LF slope. Gender was not significantly correlated with either HF or LF slope. There was a significant relationship between HF slope and birth gestational age (table 2). No other significant relationships were detected; however, the relationship between HF slope and birth weight is nearly significant.

\section{DISCUSSION}

The slope of the HF power (PNS activity) was expected to increase during and after enteral feeding; however, this was not the case. In fact, the slope of change in HF power was negative, a response contrary to what was expected with stimulation of the gut. Inhibition of SNS activity would also be expected with an enteric stimulus. In fact, the slope of change in LF power (SNS activity) decreased with feeding as anticipated. In this study, stimulation of the gut did not appear to stimulate the PNS, but there is strong evidence of SNS suppression. As seen in the graphic display (figs 1 and 2), the LF and HF slopes were both negative, suggesting that stimulation of one branch of the ANS has no effect on the

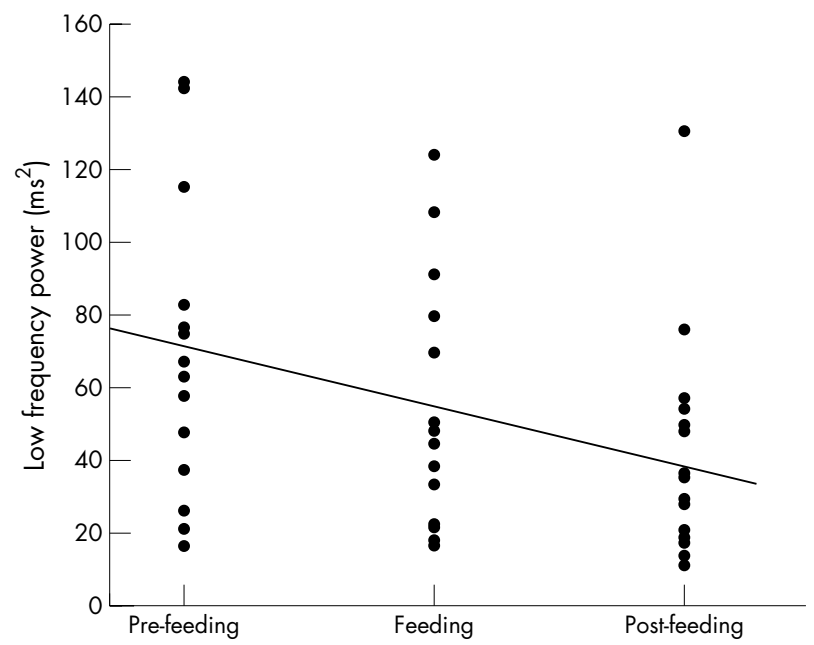

Figure 1 Average of the within subject linear growth curve models of low frequency power for 16 mechanically ventilated VLBW infants before, during, and after enteral feeding.

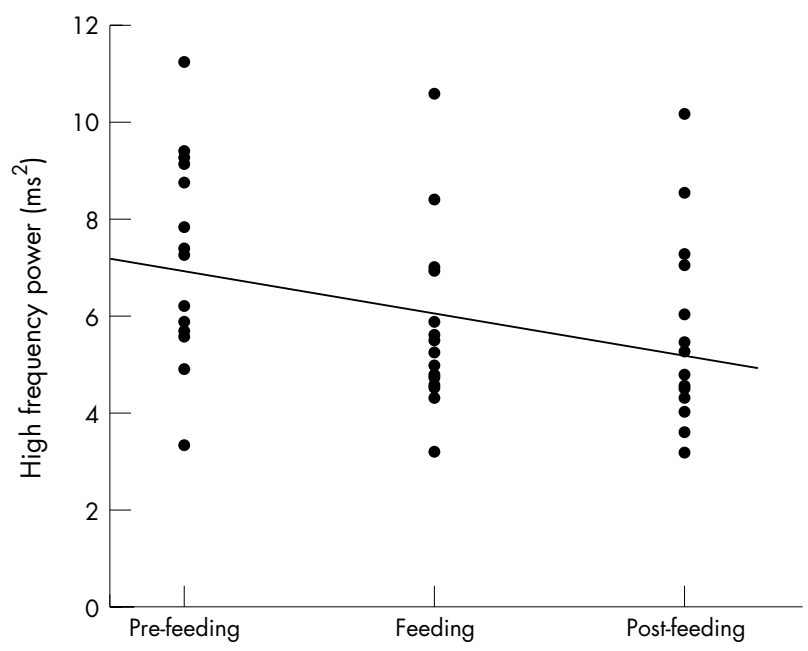

Figure 2 Average of the within subject linear growth curve models of $\mathrm{HF}$ power for 16 mechanically ventilated VLBW infants before, during, and after enteral feeding.

other branch of the ANS in these infants. These results are congruent with prior research that demonstrated autonomic imbalances in premature infants evidenced by overriding SNS activity..$^{8-10}$

The negative relationship between birth gestational age and birth weight with HF slope was another unexpected finding. This result suggests that the PNS response to feeding is reduced in the gestationally older and heavier infants and appears to be contrary to what researchers of gestationally older, healthier premature populations have found..$^{-10}$ Possible explanations for this unexpected finding could be that the gestationally older infant is able to mobilise the SNS in response to a chaotic environment in a more organised fashion. One could also speculate that these older infants who are intubated and mechanically ventilated are on constant sympathetic alert because of their degree of illness.

Although not statistically significant, the negative correlation of CGA with the slope of HF power is also contrary to what would be expected. In this study, the more gestationally mature infants exhibited a weaker PNS response. Parasympathetic activity is necessary for normal digestive function and plays a role in restorative function, growth, and development in premature infants. ${ }^{2}$ The findings in this study suggest that elements in the NICU environment may impair PNS maturation that should be occurring during the infant's hospitalisation.

\section{Limitations}

The limitations of this study include limited statistical power due to the small sample size. In addition, these data were previously acquired in a separate study thus limiting the ability to control for the NICU environment. The number of time points was constrained to three and thus we have modelled only straight line trajectories. This, in conjunction with the small sample size $(n=16)$ has limited our ability to model more complex non-linear processes of change. Finally, the NICU study site has been redesigned since these data were acquired, with significant changes in the physical environment including semiprivate rooms within the nursery, acoustic tiles, and low level lighting. With these improvements in the physical environment, a study of similar infants in these new surroundings may provide different results. 


\section{What is already known on this topic}

- The parasympathetic nervous system develops later in gestation

- The premature infant has an overriding sympathetic nervous system

\section{What this study adds}

- Promoting PNS tone is difficult to accomplish even with stimulation of the PNS

- Intubated very low birthweight infants may be on constant sympathetic alert, thus attenuating PNS development and maturation

\section{CONCLUSIONS}

Heart period power is a precise measure of ANS responses to various stimuli. Intubated VLBW infants in this exploratory study failed to demonstrate improved PNS tone in response to enteral feeding; nor did the infants' PNS tone improve with gestational age. Study of a larger cohort of VLBW infants with respiratory disease could provide the necessary statistical power to detect differences in ANS maturation and identify factors affecting the maturation process in these critically ill infants. Longitudinal research to quantify the amount of stimulus to promote ANS development in the NICU environment may help to isolate the factors inhibiting PNS maturation in these infants.

\section{Authors' affiliations \\ S L Smith, A K Doig, W N Dudley, University of Utah, Salt Lake City, UT, USA}

This study was funded by a grant from the National Heart, Lung, and Blood Institute at the National Institutes of Health (NHLBI RO1 HL7192001)

Competing interests: none declared

\section{REFERENCES}

1 Fox NA. Maturation of autonomic control in preterm infants. Dev Psychbiol 1983;16:495-504.

2 Porges SW. Cardiac vagal tone: a physiologic index of stress. Neurosci Biobehav Rev 1995; 19:225-33.

3 Massin MM, Withofs N, Maeyns K, et al. The influence of fetal and postnatal growth on heart rate variability in young infants. Cardiology 2001;95:80-3.

4 Veerappan S, Harel R, Craelius W, et al. Spectral analysis of heart rate variability in premature infants with feeding bradycardia. Pediatr Res 2000;47:659-62.

5 Jenkins JG, Reid MM, McClure BG. Study of heart rate variability in sick newborn infants. Acta Paediatr 1980;69:393-6.

6 Van Leeuwen $P$, Lange $S$, Betterman $H$, et al. Fetal heart rate variability and complexity in the course of pregnancy. Early Hum Dev 1999;54:259-69.

7 Fleisher LA, DiPietro JA, Johnson TRB, et al. Complementary and noncoincident increases in heart rate variability and irregularity during fetal development. Clin Sci 1997;92:345-9.

8 Clairambault J, Curzi-Dascalova L, Kauffmann F, et al. Heart rate variability in normal sleeping full-term and preterm neonates. Early Hum Dev 1992; 28:169-83.

9 Chatow U, Davidson S, Reichman BL, et al. Development and maturation of the autonomic nervous system in premature and full-term infants using spectral analysis of heart rate fluctuations. Pediatr Res 1995;37:294-302.

10 Sahni R, Schulze KF, Kashyap S, et al. Maturational changes in heart rate and heart rate variability in low birth weight infants. Dev Psychobiol 2000;37:73-81.

11 Burr RL, Cowan MJ. Autoregressive spectral models of heart rate variability. J Electrocardiol 1992;25(Suppl):224-33.

12 Kamath MV, Fallen EL. Power spectral analysis of HRV: a noninvasive signature of cardiac autonomic functions. Crit Rev Biomed Eng 1993;21:245-311.

13 Giddens DP, Kitney RI. Neonatal heart rate variability and its relation to respiration. J Theor Biol 1985;113:759-80.

14 Smith SL, Doig AK, Dudley WN. Characteristics of heart period variability in intubated very-low-birth-weight infants with respiratory disease. Biol Neonate 2004;86:269-74.

15 Singer JD. Using SAS PROC MIXED to fit multilevel models, hierarchical models, and individual growth models. J Educ Behav Stat 1998;24:323-55.

16 Francis DJ, Fletcher JM, Stuebing KK, et al. Analysis of change: modeling individual growth. J Consult Clin Psychol 1991;59:27-37.

17 Burchinal M, Appelbaum MI. Estimating individual developmental functions: methods and their assumptions. Child Dev 1991;62:23-43.

18 Smith SL. Physiologic stability of intubated VLBW infants during skin-to-skin care and incubator care. Adv Neonatal Care 2001;1:28-48.

19 Smith SL. Heart period variability of intubated very-low-birthweight infants during incubator care and maternal holding. Am J Crit Care 2003;12:54-64.

20 Singer JD, Willett JB. Applied longitudinal data analysis. New York: Oxford University Press, 2003.

21 Singer JD. Fitting individual growth models using SAS PROC MIXED. In: Moskowitz DS, ed. Modeling intraindividual variability with repeated measures data: Methods and applicationsMultivariate applications book series. Mahwah, NJ: Lawrence Erlbaum Associates, 2002:135-70. 